\title{
Expression of Treponema denticola Oligopeptidase B in Escherichia coli
}

\author{
Si Young Lee, ${ }^{1,2}$ J. Christopher Fenno ${ }^{2}$ \\ ${ }^{1}$ Department of Oral Microbiology, College of Dentistry, Research Institute of Oral Science, Kangnung National University, Kangnung, 201- \\ 702, Korea \\ ${ }^{2}$ Department of Biologic and Materials Sciences, School of Dentistry, University of Michigan, Ann Arbor, MI 48109-1078, USA
}

Received: 4 June 2003 / Accepted: 16 September 2003

\begin{abstract}
Treponema denticola is a small anaerobic spirochete often isolated from periodontal lesions and closely associated with periodontal diseases. This bacterium possesses a particular arginine peptidase activity (previously called "BANA-peptidase" or "trypsin-like enzyme") that is common to the three cultivable bacterial species most highly associated with severe periodontal disease. We recently reported the identification of the $o p d B$ locus that encodes the BANA-peptidase activity of $T$. denticola through DNA sequencing and mutagenesis studies. In the present study, we report expression of $T$. denticola OpdB peptidase in Escherichia coli. The opdB PCR product was cloned into pET30b and then transformed into the E. coli BL21 (DE3)/pLysS expression strain. Assays of enzymatic activities in $E$. coli containing $T$. denticola opdB showed BANA-peptidase activity similar to that of $T$. denticola. Availability of this recombinant expression system producing active peptidase will facilitate characterization of the potential role of this peptidase in periodontal disease etiology.
\end{abstract}

A specific complex of three cultivable anaerobic organisms including Treponema denticola, Porphyromonas gingivalis, and Bacteroides forsythus is associated with periodontal disease severity [11]. These bacteria express high levels of a particular peptidase activity previously designated "BANA-peptidase" or "trypsin-like enzyme" that is absent or expressed at low levels by other microbes [4]. Presence of one or more of these organisms in subgingival plaque of periodontal lesions can be determined by the ability of the plaque to hydrolyze $N-\alpha-$ benzoyl-DL-arginine-2-naphthylamide (BANA) and $N$ - $\alpha$ benzoyl-L-arginine- $p$-nitroanilide (BApNA) [5,6]. A clinical diagnostic "BANA-test" kit has been developed and tested in practice $[5,6]$. Even though the association of periodontal disease severity with these specific organisms and with high levels of BANA-hydrolyzing activity is well established, no studies have been conducted to investigate whether this enzyme activity itself has a function in periodontal disease pathogenesis.

Treponema denticola is a cultivable anaerobic spirochete often isolated from periodontal lesions. This

Correspondence to: J.C. Fenno; email: fenno@umich.edu bacterium expresses several virulence behaviors, such as adherence, cytotoxicity, iron sequestration, and locomotion, which are likely to contribute to the periodontal disease process [2]. A BANA-peptidase of T. denticola has been isolated, and its biochemical activity has been characterized [8-10]. The activity profile of the outer membrane-associated enzyme showed it to be an oligopeptidase capable of hydrolyzing ester, amide, and peptide bonds involving the carboxyl group arginine and lysine and, combined with partial peptide sequences [7], suggested that it belongs to the superfamily of prolyl oligopeptidase that includes Escherichia coli oligopeptidase B (EC 3.4.21.83). Recently, the opdB locus that encodes the BANA-peptidase activity of $T$. denticola was identified. In that study, an isogenic $T$. denticola $o p d B$ mutant constructed by allelic replacement mutagenesis demonstrated that $o p d B$ encodes all trypsinlike activity of $T$. denticola under in vitro conditions [3].

While the $o p d B$ gene and the native $\mathrm{OpdB}$ protein have been characterized, expression of this gene in $E$. coli has not been previously described. Availability of a recombinant expression system for the BANA-peptidase will facilitate further investigation of the potential role of 
opdB PCR product with $5^{\prime} \mathrm{Ndel}$ and 3 Hind III (CX230-CX232)

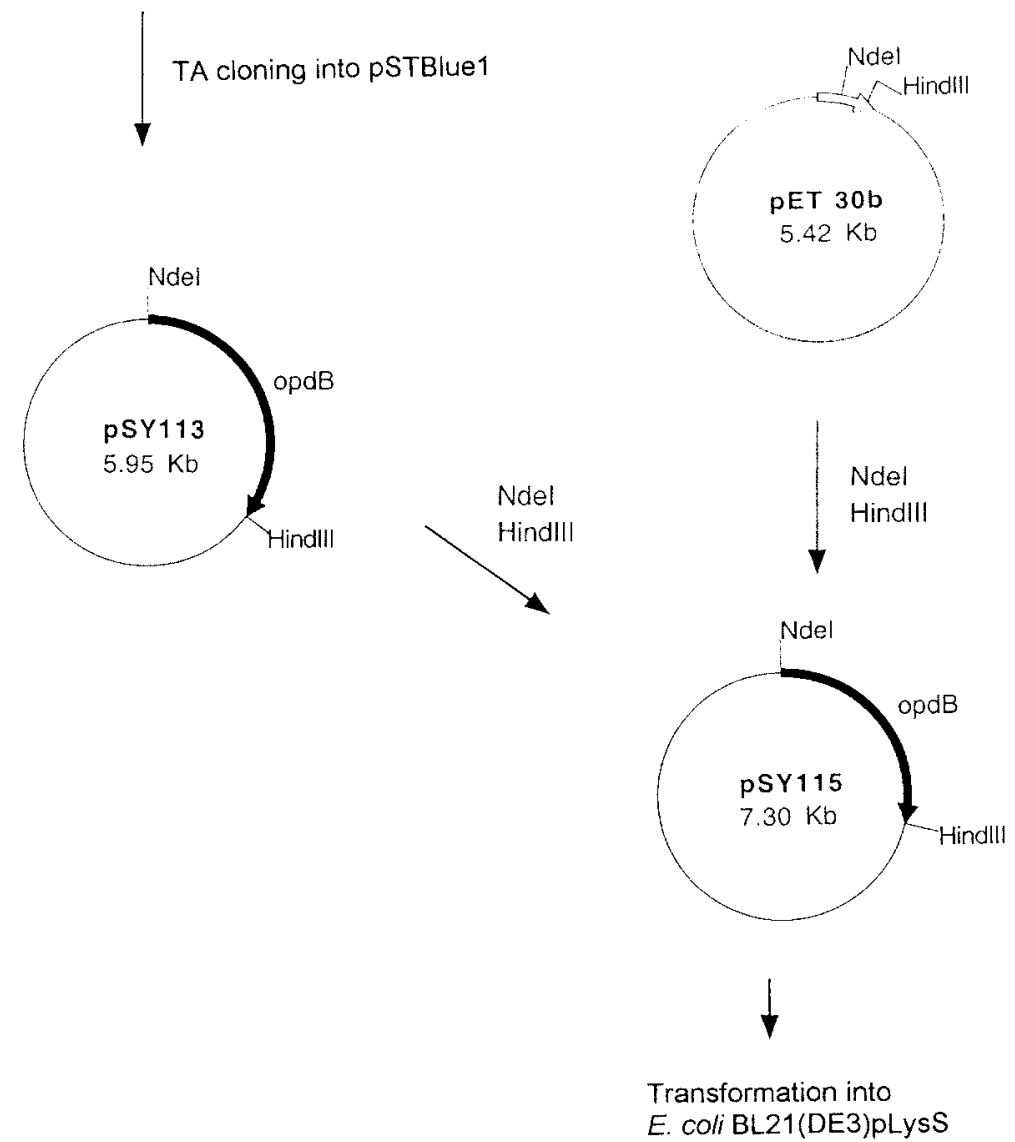

Fig 1. Strategy of cloning $o p d B$ into pET30b. The opdB PCR product was TA cloned into pSTBlue-1. The resulting plasmid pSY113 was digested with NdeI and HindIII and cloned into pET30b digested with the same set of restriction enzymes. The resulting plasmid pSY115 was transformed into $E$. coli BL21(DE3)/pLysS strain. this enzyme in the pathogenesis of $T$. denticola. In this study, we report recombinant expression of the T. denticola BANA-peptidase in E. coli.

\section{Materials and Methods}

Bacteria and culture conditions. T. denticola ATCC 35405 was grown in NOS broth as previously described [1]. Genomic DNA was prepared from $T$. denticola by using a Wizard Genomic DNA purification kit (Promega, Madison, WI, USA). E. coli JM109 and BL21 (DE3)/pLysS (Novagen, Inc., Madison, WI, USA) were used as hosts for cloning and expression, respectively. E. coli was grown on LB agar or broth medium with appropriate antibiotic selection.

Cloning into pET30b and expression. Oligonucleotide primers CX230 (5' GGA GAA ATC ATA TGA AAC AAT CTG ATT CTG AAA AGC CGC C $3^{\prime}$ ) and CX232 (5' GAT TAA ATC AAG AAC CCC GAC AAG CTT CGG GGT ACA GTG C $3^{\prime}$ ) were used to amplify a DNA fragment including $o p d B$ from $T$. denticola genomic DNA. PCR amplification of DNA was performed on a programmable thermal controller (PTC-100, MJ Research, Inc., Watertown, MA, USA). The reaction mixture $(100 \mu \mathrm{L})$ contained $0.5 \mu \mathrm{M}$ of each primer, $0.2 \mathrm{~mm}$ each of the four deoxynucleoside triphosphates, $50 \mathrm{~mm} \mathrm{KCl}, 20$ mM Tris- $\mathrm{HCl}(\mathrm{pH} 8.4), 2.5 \mathrm{~mm} \mathrm{MgCl}_{2}$, and $2.5 \mathrm{U}$ of Taq DNA polymerase (Invitrogen, Carlsbad, CA, USA). The following reaction conditions were used: annealing, $55^{\circ} \mathrm{C}$ for $30 \mathrm{~s}$; extension, $72^{\circ} \mathrm{C}$ for 2 min; and denaturation, $95^{\circ} \mathrm{C}$ for $30 \mathrm{~s}$. The cycles were repeated 30 times. PCR amplification products were cloned into TA cloning vector pSTBlue-1 by using Acceptor Vector Kits (Novagen), yielding pSY113. pSY113 was digested with NdeI and HindIII and cloned into expression vector pET30b (Novagen), yielding pSY115 (Fig. 1). Expression of $o p d B$ cloned into pET30b was performed as described in pET expression system manual book supplied by Novagen. Briefly, pSY115 was transformed into E. coli BL21(DE3)/pLysS and plated onto LB agar plate containing $30 \mu \mathrm{g} / \mathrm{mL}$ of kanamycin and $34 \mu \mathrm{g} / \mathrm{mL}$ of chloramphenicol. A single transformed colony was inoculated into LB broth containing $30 \mu \mathrm{g} / \mathrm{mL}$ of kanamycin and $34 \mu \mathrm{g} / \mathrm{mL}$ of chloramphenicol, and grown at $37^{\circ} \mathrm{C}$ on a shaking incubator until $\mathrm{OD}_{600}$ reached 0.5 . For inducing expression, isopropylthiogalactopyranoside (IPTG; Sigma, St. Louis, MO, USA) was added to a final concentration of $1 \mathrm{~mm}$. After incubating a further $2.5 \mathrm{~h}$, the bacterial suspension was centrifuged, and the bacterial pellet was stored at $-70^{\circ} \mathrm{C}$ for further use.

DNA sequencing. The DNA sequence of $o p d B$ cloned into pET30b was examined using the T7 promoter primer ( $5^{\prime}$ TAA TAC GAC TCA CTA TAG GG 3'; Novagen) to confirm the expected plasmid construct. Sequencing reactions were performed by using ABI PRISM BigDye Terminator Cycle Sequencing Kits with fluorescent-labeled dideoxynucleoside triphosphates (Applied Biosystems Inc., Foster 
A. BApNA assay

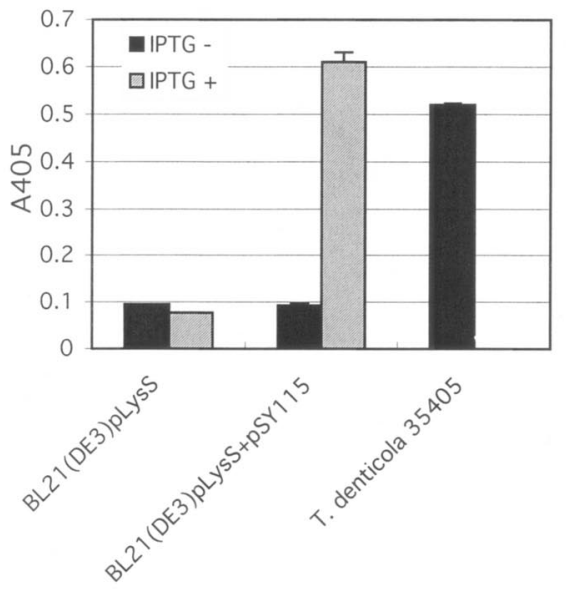

B. SAApNA assay

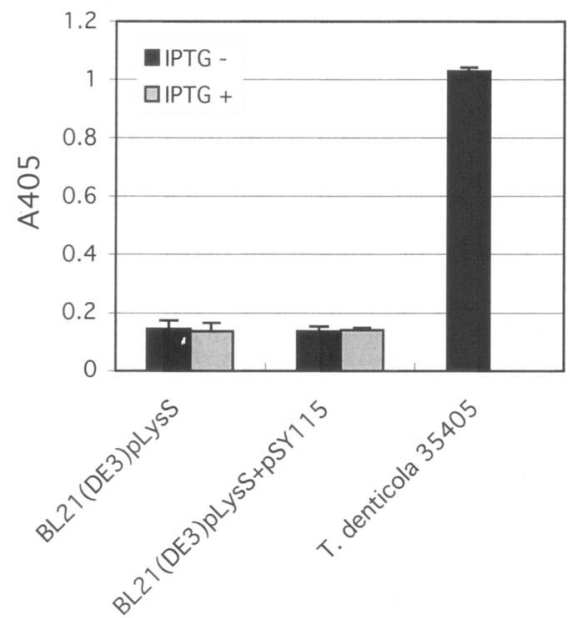

Fig 2. Enzymatic activities of E. coli BL21(DE3)/pLysS transformed with pSY115 and T. denticola ATCC 35405. (A) Trypsin-like activity measured by BApNA hydrolysis. (B) Chymotrypsin-like activity measured by SAAPF $p$ NA hydrolysis. Vertical bars show standard deviations of triplicate samples.

City, CA, USA) and a primer, according to the manufacturer's instructions. DNA sequences were resolved by using an ABI Prism 310 Genetic Analyzer (PE Applied Biosystems).

Enzymatic activity assays. Enzymatic activities of $T$. denticola and $E$. coli strains containing $T$. denticola opdB were tested by assay of hydrolysis of chromogenic substrates BApNA and succinyl-L-alanylL-prolyl-L-phenylalanine- $p$-nitroanilide (SAAPF $p$ NA; Sigma) as described previously $[3,12]$. Cultures were adjusted to an $A_{600}$ of 0.25 and then diluted 1:8 in deionized water for assays. NOS broth diluted $1: 8$ in deionized water served as a negative control.

\section{Results and Discussion}

In designing an expression system for $T$. denticola $o p d B$, we faced two significant technical issues. Firstly, in our experience recombinant expression of spirochete secreted proteins is often toxic to E. coli. Secondly, attaining expression of enzymatically active recombinant proteins can be problematic in any system. We utilized a strategy in which the opdB coding sequence was first cloned, with an engineered ATG start codon replacing the native TTG start codon, in opposite orientation to a vector-encoded promoter, yielding pSY113 (Fig. 1). From this plasmid, the $o p d B$ coding sequence was subcloned into the expression site in pET30b, such that the $o p d B$ start codon was located optimally downstream of the vector-encoded ribosome binding site and T7 RNA polymerase promoter (pSY115, Fig. 1). The DNA sequence of $o p d B$ cloned into pET30b was confirmed by sequencing (data not shown). After transfer of pSY115 to the expression host BL21(DE3)/pLysS, opdB transcription could be controlled by IPTG induction of expression of T7 RNA polymerase in this strain.
Enzymatic activities in E. coli BL21(DE3)/pLysS transformed with pSY115 (pET30b with opdB) are shown in Fig. 2A. Induction of $o p d B$ expression resulted in a high level of BApNA-hydrolyzing activity, equal that of T. denticola ATCC 35405 cultures at the same optical density. Enzyme activity in uninduced cultures was barely detectable and was indistinguishable from that of the host strain lacking pSY115. This observation is consistent with the presence of a native $o p d B$ gene in $E$. coli that is expressed at very low levels.

To confirm the specificity of the OpdB enzyme activity expressed in $E$. coli, we also tested the ability of induced cultures expressing the $T$. denticola opdB gene to hydrolyze SAAPF $p$ NA, a chromogenic substrate recognized by the T. denticola CTLP protease (dentilisin). In contrast to the high level of activity in $T$. denticola, there was no significant SAAPF $p$ NA-degrading activity in any of the E. coli strains with or without IPTG induction (Fig. 2B).

Though the BApNA hydrolyzing activity of IPTG-induced $E$. coli BL21(DE3)/pLysS transformed with pSY115 was as high as that of $T$. denticola ATCC 35405 , expression of the OpdB protein in this vector system was not very efficient. Coomassie Blue-stained SDS-PAGE of IPTG-induced E. coli BL21(DE3)/ pLysS transformed with pSY115 did not show any over-expressed protein band when compared with $E$. coli BL21(DE3)/pLysS non-transformed with pSY115 (data not shown). This is consistent with our observation in T. denticola that, though there is a significant level of OpdB enzyme activity, the OpdB protein is 
not highly expressed compared with other membraneassociated proteins [3].

In this study, we achieved recombinant expression of the $T$. denticola ATCC 35405 BANA-peptidase gene $o p d B$ in $E$. coli and demonstrated that the recombinant strain produced the specific peptidase activity at levels similar to that of $T$. denticola. Availability of a recombinant expression system that produces active OpdB peptidase will facilitate characterization of the potential role of this "BANA-peptidase" in periodontal disease etiology.

\section{ACKNOWLEDGMENTS}

The authors thank Walter Loesche for encouragement and helpful discussions. This work was supported by Kangnung National University, Korea (S.Y. Lee) and Public Health Service grant DE13565 from the National Institute of Dental and Craniofacial Research, USA (J.C. Fenno).

\section{Literature Cited}

1. Egli C, Leung WK, Müller KH, Hancock RE, McBride BC (1993) Pore-forming properties of the major 53-kilodalton surface antigen from the outer sheath of Treponema denticola. Infect Immun 61:1694-1699

2. Fenno JC, McBride BC (1998) Virulence factors of oral treponemes. Anaerobe 4:1-17

3. Fenno JC, Lee SY, Bayer CH, Ning Y (2001) The opdB locus encodes the trypsin-like peptidase activity of Treponema denticola. Infect Immun 69:6193-6200
4. Loesche WJ, Syed SA, Stoll J (1987) Trypsin-like activity in subgingival plaque. A diagnostic marker for spirochetes and periodontal disease? J Periodontol 58:266-273

5. Loesche WJ, Bretz WA, Lopatin D, Stoll J, Rau CF, Hillenburg KL, Killoy WJ, Drisko CL, Williams R, Weber HP (1990a) Multicenter clinical evaluation of a chairside method for detecting certain periodontopathic bacteria in periodontal disease. J Periodontol 61:189-196

6. Loesche WJ, Giordano J, Hujoel PP (1990b) The utility of the BANA test for monitoring anaerobic infections due to spirochetes (Treponema denticola) in periodontal disease. J Dent Res 69: $1696-1702$

7. Mäkinen KK, Mäkinen PL (1996) The peptidolytic capacity of the spirochete system. Med Microbiol Immunol 185:1-10

8. Mäkinen KK, Chen CY, Mäkinen PL, Ohta K, Loesche WJ (1990) The benzoylarginine peptidase from Treponema denticola (strain ASLM), a human oral spirochaete: evidence for active-site carboxyl groups. Mol Microbiol 4:1413-1417

9. Mäkinen KK, Mäkinen PL, Loesche WJ, Syed SA (1995) Purification and general properties of an oligopeptidase from Treponema denticola ATCC 35405-a human oral spirochete. Arch Biochem Biophys 316:689-698

10. Ohta K, Mäkinen KK, Loesche WJ (1986) Purification and characterization of an enzyme produced by Treponema denticola capable of hydrolyzing synthetic trypsin substrates. Infect Immun 53:213-220

11. Socransky SS, Haffajee AD, Cugini MA, Smith C, Kent RL Jr (1998) Microbial complexes in subgingival plaque. J Clin Periodontol 25:134-144

12. Uitto V-J, Grenier D, Chan EC, McBride BC (1988) Isolation of a chymotrypsinlike enzyme from Treponema denticola. Infect Immun 56:2717-2722 